\title{
Evaluation of the surgical difficulty in lower third molar extraction
}

\author{
José Barreiro-Torres ${ }^{1}$, Marcio Diniz-Freitas ${ }^{1}$, Lucía Lago-Méndez ${ }^{1}$, Francisco Gude-Sampedro ${ }^{2}$, José-Ma- \\ nuel Gándara-Rey ${ }^{3}$, Abel García-García ${ }^{4}$ \\ ${ }^{1}$ Oral Surgery and Oral Medicine Unit, Dental school, University of Santiago de Compostela, Spain \\ ${ }^{2}$ Staff, Clinical Research Unit, Complejo Hospitalario Universitario de Santiago de Compostela, Spain \\ ${ }^{3}$ Professor of Oral Medicine, School of Dentistry, University of Santiago de Compostela, Spain \\ ${ }^{4}$ Professor of Oral Surgery, School of Dentistry, University of Santiago de Compostela, Spain. Head of Section, Department of \\ Maxillofacial Surgery, Complejo Hospitalario Universitario de Santiago de Compostela, Spain
}

Correspondence:

Facultad de Odontología

c/ Entrerrios $s / n$

15782 Santiago de Compostela

A Coruña, Spain

pepe.barreiro@gmail.com

Received: 02/07/2009 Accepted: 21/02/2010
Barreiro-Torres J, Diniz-Freitas M, Lago-Méndez L, Gude-Sampedro F, Gándara-Rey JM, García-García A. Evaluation of the surgical difficulty in lower third molar extraction. Med Oral Patol Oral Cir Bucal. 2010 Nov $1 ; 15(6): \mathrm{e} 869-74$.

http://www.medicinaoral.com/medoralfree01/v15i6/medoralv15i6p869.pdf

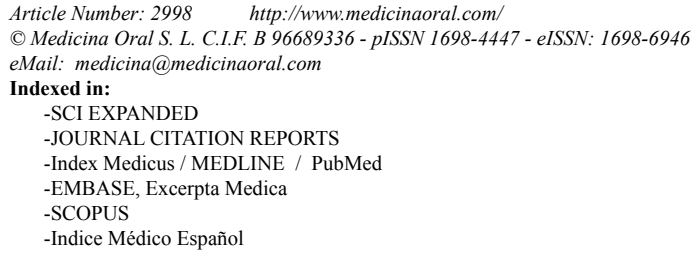

\begin{abstract}
The ability to predict the surgical difficulty of lower third molar extraction facilitates the design of treatment plans by minimizing complications and improving the preparation of patients and assistants in terms of the postoperative management of inflammation and pain. The aims of this study were to evaluate the value of panoramic radiographs in predicting lower third molar extraction difficulty and technique and to determine if the experience of the practitioner had any influence on this predictive ability.

Fourteen dental practitioners with varying levels of experience evaluate the difficulty of lower third molar extraction in a group of patients using a 100 -mm visual analog scale (VAS) and a modified version of a surgical difficulty scale. The results were then compared to postoperative scores calculated using the same scale.

A tendency to underestimate the difficulty of procedures that was more pronounced in observers with greater levels of experience was observed. A low level of agreement between preoperative and postoperative evaluations using the surgical difficulty scale as well as an association between difficulty assessed preoperatively using the VAS and difficulty assessed postoperatively using the surgical difficulty scale was also found.

The use of panoramic radiographs does not allow practitioners to accurately predict lower third molar extraction difficulty and technique, regardless of their level of experience.
\end{abstract}

Key words: Lower third molar, oral surgery, surgical difficulty. 


\section{Introduction}

Although the extraction of impacted lower third molars is a common dental procedure, it can present difficulties. Several classification systems have been established to estimate the surgical difficulty of removing lower third molars but they have proven to be of little clinical use $(1,2)$. These systems are primarily based on the preoperative assessment of panoramic radiographs but other factors such as demographic and operative variables have also been analyzed (3-9).

The ability to predict the surgical difficulty of lower third molar extraction is essential when designing a treatment plan in that it helps to assess the competence of the dental practitioner for the particular operation, minimize complications (10), and optimize the preparation of the patient and assist in terms of the postoperative management of inflammation and pain. This ability to predict the difficulty of third molar extraction has been found to vary according to the experience of the practitioner with the procedure (11).

The aim of this study was to evaluate the value of panoramic radiographs in the prediction of lower third molar extraction difficulty and technique and to determine if this is influenced by the experience of the practitioner (primary care dentist [PCD], dental oral surgery specialist [DOS], and maxillofacial surgeon [MS].

\section{Material and Methods}

\section{Patient Selection}

We studied 80 lower third molar extractions performed in randomly selected patients who visited the Master' of Oral Medicine, Oral Surgery and Implantology (MOMOSI) at the dental school of the University of Santiago de Compostela in Spain between February 2008 and April 2009. The first of every fifth patient was atended on the days on which lower third molar extractions were performed, was included in the study.

A complete clinical history was taken at the first visit, with collection of the following information: patient age and sex; identification of the molar to be removed and reason for removal; level of impaction (totally covered by bone, totally covered by soft tissue, partially covered by soft tissue, or completely erupted); relative depth and space for eruption according to the Pell-Gregory classification; angle according to Winter's classification $(12,13)$; and the presence of associated symptoms and/or diseases. Informed consent was obtained from all patients, each of whom agreed to be treated and to participate in the study.

Data collection

Panoramic radiographs were scaned for each patient at a resolution of 150 ppi (Agfalook 32 V3. 60.0, Arcus 1200 ) and then shown to 14 dental practitioners with different levels of surgical experience. These included 2 primary care dentists from the Galician Public Health
Care System (SERGAS), 10 dental oral surgery specialist from the master, and 2 maxillofacial surgeons from the Complejo Hospitalario Universitario de Santiago de Compostela in Galicia (CHUS), Spain. For each case, each observer decided whether or not lower third molar extraction was indicated, predicted the difficulty of the operation, and recorded which technique they would use.

\section{Preoperative evaluation of surgical difficulty}

Surgical difficulty was evaluated preoperatively using a version of the Parant scale modified by García-García et al. (14) (Table 1). This scale defines 4 levels of difficulty depending on the surgical maneuvers required for the extraction of lower third molars: I: simple extraction; II: extraction requiring ostectomy; III; extraction requiring ostectomy and coronal section; and IV: complex extraction (root section). Each of the observers also predicted surgical difficulty using a visual analog scale (VAS) of $100 \mathrm{~mm}$, where 0 represented the lowest imaginable difficulty, and 100, the greatest imaginable difficulty.

Surgical technique

All interventions were performed by postgraduate students from the MOMOSI. All surgeries were performed under local anesthesia by nerve-block anesthesia of the inferior alveolar nerve, lingual nerve and buccal nerve, with two $1.8-\mathrm{mL}$ capsules of $4 \%$ articaine with epinephrine 1:200,000 (Ultracain; Normon, Madrid, Spain). In cases where an elevator and/or forceps were not effective, a mucoperiosteal flap was raised, generally by an incision distal to the lower second molar along the anterior border of the ascending ramus of the mandible, with mesial releasing incision in this molar. Ostectomy and tooth or root sectioning were performed where necessary using a low-speed round $\mathrm{n}^{\circ} 8$ tungsten carbide bur. The area was irrigated with saline solution and curettage of granulation tissue was performed. The wound was sutured with 3/0 silk sutures (Mersilk; Ethicon, Livingston, United Kingdom), and a folded gauze was applied over the surgical wound to achieve compression and adequate hemostasis. The sutures were removed a week after the operation. Patients were also given appropriate instructions and recommendations regarding the postoperative recovery period.

Table 1. Surgical difficulty scale.

\begin{tabular}{|c|l|}
\hline \multicolumn{2}{|c|}{ Parant surgical difficulty scale modified by Garcia-Garcia } \\
\hline Type & \multicolumn{1}{|c|}{ Technique } \\
\hline I & Simple extraction \\
II & Extraction requiring ostectomy \\
III & Extraction requiring ostectomy and coronal section \\
IV & Complex extraction (root section) \\
\hline
\end{tabular}


Each patient was prescribed an antibiotic (amoxicillin: $2 \mathrm{~g} 1$ hour before the operation $+1 \mathrm{~g}$ every 8 hours for 7 days), an anti-inflammatory analgesic (iboprufen $600 \mathrm{mg}$; 1 tablet every 8 hours for 4-5 days, starting immediately after the operation), and an antiseptic (Clorhexidin $0.12 \%$ mouthwash 3 times a day for 7 days, starting the day after the operation).

Postoperative evaluation of surgical difficulty

At the end of the surgery, the surgeon recorded the technique used to extract the tooth on a special form. With this information, the observers rated surgical difficulty once again using the modified Parant scale (14). The duration of each operation (from the first extraction maneuver to the completion of the last suture) was also noted.

\section{Statistical analysis}

Qualitative variables were expressed as absolute frequencies and percentages and quantitative variables as means (SD). The weighted kappa statistic (15) was used to assess the level of agreement between preoperative and postoperative evaluations of surgical difficulty. Analysis of variance was used to test for an association between the VAS and the surgical difficulty scale. A p value of less than .05 was considered statistically significant.

Version 15.0 of the statistical software package SPSS for Microsoft Windows was used to analyze all data.

\section{Results}

\section{Characteristics of sample}

Eighty lower third molars were extracted from 66 patients (23 men and 43 women) during the study period. The ratio of left to right molars was 1:1 and the mean (SD) age of the patients was $25.4 \pm 6.6$ years (range, 16 46 years). The most common indication for extraction was pericoroniritis $(n=32,40.2 \%)$, followed by proph- ylaxis $(\mathrm{n}=25,31.3 \%)$. Forty $(50 \%)$ of the lower third molars were partially erupted, $19(23.8 \%)$ were totally erupted, 15 (18.7\%) were partially covered by bone, and $6(7.5 \%)$ were totally impacted.

On the basis of Winter's classification, 29 molars (36.3\%) were vertical, 25 (31.3\%) were mesioangular, 14 (17.5\%) were horizontal, 10 (12.5\%) were distoangular, and $2(2.5 \%)$ were inverted. According to the PellGregory classification, 37 (46.3\%) were in position IIA, $14(17.5 \%)$ in position IIB, $13(16.3 \%)$ in position IA, $9(11.3 \%)$ in position IIIA, $3(3.8 \%)$ in position IB, 3 (3.8\%) in position IIIB, and $1(1.3 \%)$ in position IIC.

The group of maxillofacial surgeons advised that extraction was indicated in $76.3 \%$ of the cases. The respective percentages for the dental oral surgery specialist and primary care dentists were $78.3 \%$ and $99.4 \%$.

Preoperative evaluation of difficulty

Figure 1 shows the mean percentage of extractions classified according to surgical difficulty by the different groups of observers. On comparing these results with those for surgical difficulty estimated postoperatively, we observed a tendency towards underestimation.

The mean (SD) difficulty score for the 80 extractions as assessed using the VAS was 30.8 (15.4) for the maxillofacial surgeons, 43.7 (22.7) for the primary care dentists, and 52.1 (12.6) for the dental oral surgery specialist.

Postoperative evaluation of surgical difficulty

Postoperatively, $13(16.3 \%)$ of the extractions were classified as type I, $25(31.3 \%)$ as type II, $18(22.5 \%)$ as type III, and $24(30 \%)$ as type IV (Fig. 1). Three (3.8\%) of the extractions were performed in less than 10 minutes, 20 (25\%) within 10 to 19 minutes, $22(27.5 \%)$ within 20 to 29 minutes, $16(20 \%)$ within 30 to 39 minutes, and 19 (23.8\%) in over 40 minutes.

The level of agreement (measured using the weighted kappa statistic) between preoperative evaluation of dif-

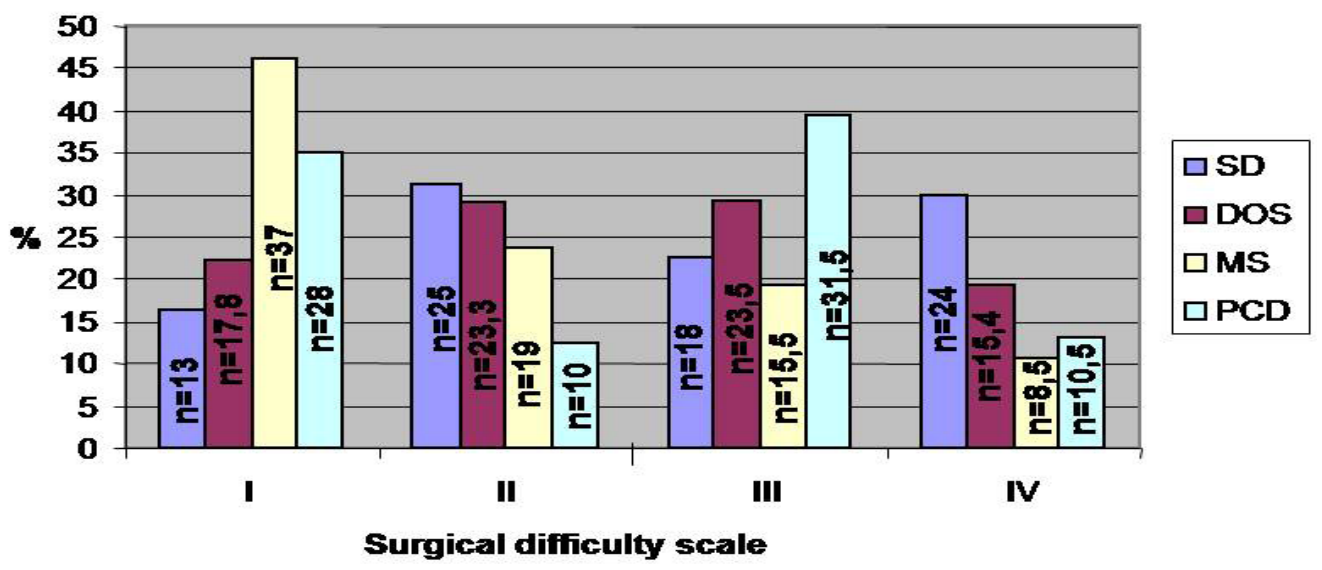

Fig. 1. Percentage of total extractions classified by the groups of observers prior to surgery as type I, II, III, or IV. Relationship between results and postoperative surgical difficulty scale. 
Table 2. Level of agreement between preoperative evaluation of surgical difficulty and postoperative evaluation for each of the observers using the weighted kappa statistic.

\begin{tabular}{|c|c|c|c|}
\hline Surgeon & Agreement \% & Kappa & Sign. \\
\hline OS1 & 67 & $0.183 \pm 0.080$ & 0.011 \\
\hline OS2 & 75.4 & $0.356 \pm 0.074$ & 0.000 \\
\hline OS3 & 74.1 & $0.328 \pm 0.077$ & 0.000 \\
\hline OS4 & 68.3 & $0.212 \pm 0.077$ & 0.002 \\
\hline OS5 & 72 & $0.355 \pm 0.069$ & 0.000 \\
\hline OS6 & 76.2 & $0.386 \pm 0.072$ & 0.000 \\
\hline OS7 & 75 & $0.371 \pm 0.079$ & 0.000 \\
\hline OS8 & 75.4 & $0.358 \pm 0.078$ & 0.000 \\
\hline OS9 & 78.3 & $0.443 \pm 0.078$ & 0.000 \\
\hline OS10 & 74.1 & $0.363 \pm 0.073$ & 0.000 \\
\hline MS1 & 69.5 & $0.332 \pm 0.070$ & 0.000 \\
\hline MS2 & 67.5 & $0.243 \pm 0.060$ & 0.000 \\
\hline PCD1 & 66.6 & $0.160 \pm 0.074$ & 0.015 \\
\hline PCD2 & 67 & $0.245 \pm 0.066$ & 0.000 \\
\hline
\end{tabular}

ficulty based on panoramic radiographs and postsurgical evaluation using the surgical difficulty scale for the different observers is shown in Table 2.

As can be seen, the weighted kappa statistic was less than 0.40 for all the observers except one oral surgeon, indicating a weak level of agreement between subjective, preoperative evaluation and objective, postoperative evaluation. The minimum and maximum kappa values for the different groups of observers were, respectively: 0.243 and 0.332 for the maxillofacial surgeons, 0.183 and 0.443 for the dental oral surgery specialist, and 0.160 and 0.245 for the primary care physicians.

Predictive ability, i.e. the number of cases in which preoperative and postoperative evaluation of difficulty coincided, was $38.7 \%$ for the maxillofacial surgeons, $45.1 \%$ for the dental oral surgery specialist, and $31.9 \%$ for the primary care dentists.

As can be seen in figure 2, statistically significantly association $(\mathrm{P}<.05)$ between the VAS score and the postoperative difficulty rating, was detected for the different groups of observers.

\section{Discussion}

To successfully evaluate the difficulty of lower third molar extraction prior to surgery, clinical and radiologic findings must be taken into account. Not only does this help to correctly plan the operation, but it also increases patients' level of satisfaction with the treatment received.

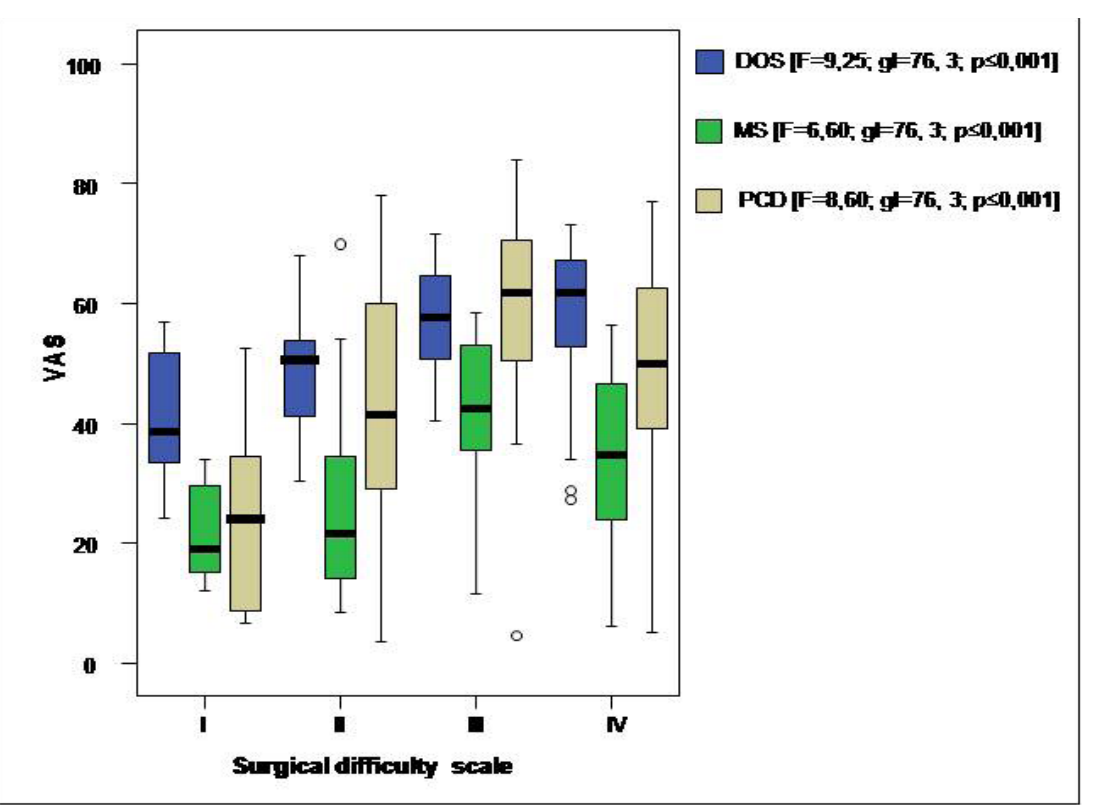

Fig. 2. Box and whisker plot showing level of agreement between preoperative estimation of surgical difficulty using the visual analog scale and postoperative estimation using a modified surgical difficulty scale for each of the groups of observers. 
Several authors have attempted to evaluate this difficulty on the basis of the position of the molar in panoramic radiographs $(12,16)$ but it has since been demonstrated that these indexes are not reliable for this purpose $(1,2)$. Other variables have also been studied, including surgical variables (such as the extracted tooth, the surgeon, and the level of impaction) and demographic variables (such as age, weight, degree of mouth opening, and cheek flexibility) $(3,9)$, with some of these factors found to have an influence on surgical difficulty. Because analyzing all of these parameters for each extraction would be very costly, Yuasa et al. (8) proposed using a simpler index based on 3 factors: the depth of the third molar in the mandible, the relationship with the ramus/space available, and root width.

In previous studies $(1,14)$, our group used a modified surgical difficulty scale to classify the difficulty of lower third molar extraction postoperatively. We consider the scale to be a reliable, consistent measure of surgical difficulty and thus believe it can be considered a gold standard test as it has been found to be significantly associated with surgery time (1). In the present study, we have assessed the value of this scale in predicting surgical difficulty based on the observation of panoramic radiographs prior to surgery. Our results indicate that it is difficult to predict surgical technique on the basis of panoramic radiographs, regardless of the level of experience of the practitioner. The level of agreement between preoperative and postoperative evaluation of extraction difficulty was slightly higher for dental oral and maxillofacial surgeons than for primary care dentists, possibly because the surgeons are more familiar with these procedures (17). The level of agreement was highest for the dental oral surgery specialist group, probably because, although they have less experience than maxillofacial surgeons, they would have been better trained to predict the technique used (based on their own skills), as the extractions in the study were performed by oral themselves. On comparing the mean preoperative difficulty scores for each of the 3 observer groups with the postoperative scores (Fig. 1), we observed a tendency to underestimate difficulty in all 3 groups. This observation is in agreement with reports from several studies $(6,7)$. The level of underestimation was most pronounced in the group with the greatest level of experience, the maxillofacial surgeons. This same trend was observed in the mean difficulty scores calculated according to the VAS for the different groups of observers (Fig. 2). FerrúsTorres et al. (11) found that residents with low levels of experience considered lower third molar extractions to be more complicated and tended to opt for surgery in more cases.

Macluskey et al. (18) found that predictive ability of surgical difficulty were accurate in $96 \%$ of cases for specialist surgical dentists from a private practice com- pared to in $66 \%$ of cases for dental hospital staff, with no association detected between predictive ability and level of experience. Predictive ability in our study was highest for the group of dental oral surgery specialist, followed by the maxillofacial surgeons and the primary care dentist, respectively, but the values in all cases were considerably lower than those reported by Macluskey et al. (18).

On analyzing the estimation of surgical difficulty using the VAS, we saw that there was a tendency to rate extractions that required a more complicated surgical technique as more difficult. These data should, however, be analyzed with caution because, as we can see in (Fig. 2), the mean results for the different groups are very different, and in addition, we are comparing a VAS with a surgical difficulty scale.

Regardless of level of experience, none of the practitioners in our study were able to accurately predict the difficulty of extraction on the basis of a panoramic radiograph. This finding supports reports by Susarla et al. $(5,6)$, who stated that surgical experience did not seem to exert a significant influence on a surgeon's ability to accurately predict surgical difficulty. They also mentioned that predictive ability improved with surgical experience. Several articles have discussed the difficulty of estimating surgical difficulty using panoramic radiographs (19) and it has been suggested that this can be done best intraoperatively (20).

In conclusion, in the present study we found a low level of agreement between preoperative evaluation of lower third molar extraction difficulty based on assessment of panoramic radiographs and a modified surgical difficulty scale compared to postoperative evaluation. Finally, the level of experience of the observers did not seem to exert a significant influence on the results.

\section{References}

1. Diniz-Freitas M, Lago-Méndez L, Gude-Sampedro F, SomozaMartin JM, Gándara-Rey JM, García-García A. Pederson scale fails to predict how difficult it will be to extract lower third molars. Br J Oral Maxillofac Surg. 2007;45:23-6.

2. García AG, Sampedro FG, Rey JG, Vila PG, Martin MS. PellGregory classification is unreliable as a predictor of difficulty in extracting impacted lower third molars. Br J Oral Maxillofac Surg. 2000;38:585-587.

3. Akadiri OA, Obiechina AE. Assessment of difficulty in third molar surgery-a systematic review. J Oral Maxillofac Surg. 2009;67:771-4.

4. Gbotolorun OM, Arotiba GT, Ladeinde AL. Assessment of factors associated with surgical difficulty in impacted mandibular third molar extraction. J Oral Maxillofac Surg. 2007;65:1977-83.

5. Susarla SM, Dodson TB. Estimating third molar extraction difficulty: a comparison of subjective and objective factors. J Oral Maxillofac Surg. 2005;63:427-34.

6. Susarla SM, Dodson TB. How well do clinicians estimate third molar extraction difficulty? J Oral Maxillofac Surg. 2005;63:191-9.

7. Renton T, Smeeton N, McGurk M. Factors predictive of difficulty of mandibular third molar surgery. Br Dent J. 2001;190:607-10.

8. Yuasa H, Kawai T, Sugiura M. Classification of surgical difficul- 
ty in extracting impacted third molars. Br J Oral Maxillofac Surg. 2002;40:26-31.

9. Santamaria J, Arteagoitia I. Radiologic variables of clinical significance in the extraction of impacted mandibular third molars. Oral Surg Oral Med Oral Pathol Oral Radiol Endod. 1997;84:469-73.

10. Contar CM, de Oliveira P, Kanegusuku K, Berticelli RD, Azevedo-Alanis LR, Machado MA. Complications in third molar removal: a retrospective study of 588 patients. Med Oral Patol Oral Cir Bucal. 2010;15:e74-8.

11. Ferrús-Torres E, Gargallo-Albiol J, Berini-Aytés L, Gay-Escoda C. Diagnostic predictability of digital versus conventional panoramic radiographs in the presurgical evaluation of impacted mandibular third molars. Int J Oral Maxillofac Surg. 2009;38:1184-7.

12. Koerner KR. The removal of impacted third molars. Principles and procedures. Dent Clin North Am. 1994;38:255-78.

13. Almendros-Marqués N, Alaejos-Algarra E, Quinteros-Borgarello M, Berini-Aytés L, Gay-Escoda C. Factors influencing the prophylactic removal of asymptomatic impacted lower third molars. Int J Oral Maxillofac Surg. 2008;37:29-35.

14. Garcia Garcia A, Gude Sampedro F, Gandara Rey J, Gallas Torreira M. Trismus and pain after removal of impacted lower third molars. J Oral Maxillofac Surg. 1997;55:1223-6.

15. Landis JR, Koch GG. An application of hierarchical kappa-type statistics in the assessment of majority agreement among multiple observers. Biometrics. 1977;33:363-74.

16. Koerner KR. The removal of impacted third molars. Principles and procedures. Dent Clin North Am. 1994;38:255-78.

17. Hazelkorn HM, Macek MD. Perception of the need for removal of impacted third molars by general dentists and oral and maxillofacial surgeons. J Oral Maxillofac Surg. 1994;52:681-6.

18. Macluskey M, Slevin M, Curran M, Nesbitt R. Indications for and anticipated difficulty of third molar surgery: a comparison between a dental hospital and a specialist high street practice. Br Dent J. 2005;199:671-5.

19. Edwards DJ, Brickley MR, Horton J, Edwards MJ, Shepherd JP. Choice of anaesthetic and healthcare facility for third molar surgery. Br J Oral Maxillofac Surg. 1998;36:333-40.

20. Chandler LP, Laskin DM. Accuracy of radiographs in classification of impacted third molar teeth. J Oral Maxillofac Surg. 1988;46:656-60. 